\title{
Percepción del servicio del colaborador interno y actitudes hacia el conflicto organizacional en instituciones del sector público de Lima Metropolitana
}

\section{Perception of the service of the internal collaborator and attitudes towards the organizational conflict in institutions of the public sector of Metropolitan Lima}

\author{
M. T. Paredes, H. M. Hernández, C. E. Malaver y A. R. Chung
}

Recibido: junio 19 de 2020 - Aceptado: diciembre 31 de 2021

\begin{abstract}
Resumen - La mayor fortaleza que tienen las organizaciones frente a entornos inestables y cambiantes, son sus colaboradores internos, de ellos depende la calidad que se brinde al cliente externo. A la vez las relaciones laborales, caracterizadas por la interacción son una fuente constante de conflictos, los que deben anticiparse o intervenir a tiempo para que o desaparezcan o se conviertan en funcionales. Por ello se investigó la relación entre dos variables psicosociales, la percepción del servicio del usuario interno y las actitudes hacia el conflicto organizacional en los usuarios internos de instituciones públicas, asumiendo que son inversas entre sí, de manera global y entre sus dimensiones. Se emplearon dos escalas tipo Likert, válidas y confiables, a 303 participantes bajo consentimiento informado. Mediante análisis confirmatorio se generó un modelo en el cual se grafica la relación entre las dimensiones de cada variable. Se confirmó la relación inversa global y entre las dimensiones, excepto para

${ }^{1}$ Producto derivado del proyecto de investigación "Percepción del servicio del colaborador interno y actitudes hacia el conflicto organizacional en instituciones del sector público de lima metropolitana". Presentado por el Grupo de Investigación Vínculos interdependientes y contextos medio ambientales saludables en el trabajo, de la facultad de Psicología, de la Universidad Nacional Mayor de San Marcos.

M. T. Paredes, Universidad Nacional Mayor de San Marcos, Lima, Perú, mparedest@unmsm.edu.pe.

H. M. Hernández, Universidad Nacional Mayor de San Marcos, Lima, Perú, hhernandezv@unmsm.edu.pe.

C. E. Malaver, Universidad Nacional Mayor de San Marcos, Lima, Perú, cmalavers@unmsm.edu.pe.

A. R. Chung, Universidad Nacional Mayor de San Marcos, Lima, Perú, achungp@unmsm.edu.pe.
\end{abstract}

Como citar este artículo: Paredes, M. T., Hernández, H. M., Malaver, C. E., y Chung, A. R. Percepción del servicio del colaborador interno y actitudes hacia el conflicto organizacional en instituciones del sector público de Lima Metropolitana, Entre Ciencia e Ingeniería, vol. 15, no. 30, pp. 71-81, juliodiciembre 2021. DOI: https://doi.org/10.31908/19098367.1704.

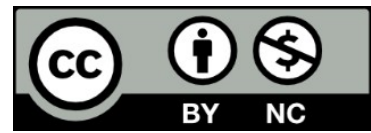

Attribution-NonCommercial 4.0 Intenational (CC By-NC 4.0) valoración de su labor y conflicto interiorizado. Los resultados encontrados posibilitan diseñar estrategias para gestionar funcionalmente el conflicto.

Palabras clave- Conflicto organizacional, Percepción al servicio del usuario interno, Conflicto organizacional por escasez, Conflicto organizacional emergente, Conflicto organizacional interiorizado.

Abstract - The greatest strength that organizations have in the face of unstable and changing environments are their internal collaborators, the quality that is provided to the external client depends on them. At the same time, labor relations, characterized by interaction, are a constant source of conflicts, which must be anticipated or intervened in time so that they either disappear or become functional. For this reason, the relationship between two psychosocial variables, perception of the service of the internal user and attitudes towards the organizational conflict in the internal users of public institutions was investigated, assuming that they are inverse to each other, globally and between their dimensions. Two valid and reliable Likert-type scales were used in 303 participants with informed consent. Through confirmatory analysis, a model was generated in which the relationship between the dimensions of each variable is plotted. The global inverse relationship and between the dimensions was confirmed, except for assessing their work and internalized conflict. The results found make it possible to design strategies to manage the conflict functionally.

Keywords - Organizational conflict, Perception at the service of the internal user, Organizational conflict by scarcity, Emerging organizational conflict, Internalized organizational conflict.

\section{INTRODUCCIÓN}

$\mathrm{L}$ A Agenda 2030 para el desarrollo sostenible plantea en su octavo objetivo, la promoción de un crecimiento económico sostenido e inclusivo, así como entre una de sus metas señala la promoción del trabajo seguro en un entorno laboral sin riesgos para todos los trabajadores [1]. Demanda de dichos contextos saludables que todo directivo debe lograr con el funcionamiento de prácticas de gestión en la multiplicidad 
de conflictos que suelen suscitarse, entre ellos, producidos en las relaciones socio laborales y evitar que repercutan negativamente en la organización [2].

En esta línea el presente estudio, se aboca hacia uno de los principales activos por constituirse en el valor vital de una organización, sus colaboradores internos, quienes ejercen a su vez influencia a la percepción de valor del cliente externo, independiente de su jerarquía y funciones que realicen, cuyo comportamiento tiene impacto en la rentabilidad de las empresas [3], por ser relevante aproximarse al comportamiento de la institución que proporcione servicios, estudiar la percepción del colaborador en su doble rol de proveedor o consumidor de servicio o bien para su actividad laboral [4] [5] y su aproximación al conflicto organizacional, en la medida que la existencia de este, como conducta generada en el proceso de interacción, puede ser funcional o disfuncional, por ende afectar positiva o negativamente en el desarrollo de las tareas $\mathrm{y} / \mathrm{o}$ en el establecimiento de las relaciones [6].

El que, como conducta generada en el proceso de interacción por deficiencias en la estructura y gestión institucional, lo que, no impide la presencia de conflictos intraindividuales que pueden ser originados por características de personalidad o circunstancias personales [7], con implicancia mayor cuando el usuario interno participa en actividades incongruentes a sus necesidades e intereses o ante la existencia de diferentes perspectivas, aunado por la sobrepresión o auto-presión en tareas, condición que agudiza el conflicto organizacional al estar asociado a una interdependencia funcional $u$ otras fuentes de conflictos que son la incompatibilidad por valores, creencias y metas [8].

Así, el conocimiento y valoración de la relevancia del rol que cumple el usuario interno para alcanzar los objetivos organizacionales dirigidos hacia el crecimiento sostenido de la institución, y la implicancia del desarrollo del colaborador como persona y factor clave en la productividad, competitividad y rentabilidad de la organización, se pretende describir e identificar la relación entre la percepción del servicio del colaborador interno y las actitudes hacia el conflicto organizacional en instituciones del sector público, la que, por su naturaleza opositora entre el reconocimiento del servicio recibido y las respuestas de agresión o imposición, debe resultar en una relación inversa en los ambientes laborales. Para esta constatación se presenta la revisión del arte de la temática de la investigación, luego la metodología, la descripción de los instrumentos construidos por los autores para medir las variables de estudio y la comprobación de las hipótesis, al final se analizan los resultados obtenidos y se concluye.

\section{MARCO TEÓRICO}

\section{A. Percepción del colaborador interno}

Actualmente las organizaciones actúan en un contexto de incertidumbre altamente competitivo, que conlleva a que éstas se propongan nuevas formas de organización a fin de facilitar la interacción entre los colaboradores internos promoviendo el liderazgo compartido [9], afrontando retos, la incertidumbre, actuando proactivamente generando cambios, innovar con calidad para captar y fidelizar clientes e incrementar su rentabilidad; por lo cual es importante atender a la calidad de servicio que se brinde al cliente interno, ya que repercutirá en la satisfacción del cliente externo final, así alcanzó dicha orientación una mayor relevancia a partir de la década de los 80 [10] al no centrarse exclusivamente en las expectativas de los directivos, sino invirtiéndose la pirámide, redireccionándose a atender las necesidades e intereses de los clientes [11], lo que conllevó a arribar que todos son clientes para una institución o empresa, sea aquellos que adquieren bienes o servicios considerados clientes externos como los que trabajan para ella, que se constituyen en clientes internos o usuarios internos.

Los usuarios internos son aquellos trabajadores o colaboradores que realizan actividades operativas, administrativas o ejecutivas, siendo proveedores o receptores de insumos tangibles o intangibles, que requieren o necesitan utilizar para alguna de sus etapas o fases del producto que laboran [4].

La reputación de la excelencia de los productos de una entidad, se cimienta en que todos sus colaboradores internos forman parte de la estrategia de servicio, aun cuando no trabajen directamente con el cliente externo, ya que pueden estar proveyéndole servicio o insumo a un colaborador que si lo hace; así cada uno de los usuarios internos tiene un efecto en la satisfacción del usuario externo final [10], aun cuando no trabajen directamente con el cliente externo, ya que pueden estar proveyéndole servicio o insumo a un colaborador que si lo hace [10]

En dicho proceso los usuarios internos forman su percepción hacia el servicio que se brindan entre sí, al servicio de la institución, independientemente de si son jefes, pares o subordinados [5]. La percepción define así su papel como un mediador clave del comportamiento al interior de la organización, en virtud a categorías aprendidas y al establecimiento de establecer comparaciones en tanto usuario interno entre sus expectativas y el servicio otorgado percibido; entre lo que pueda conseguir y el mínimo adecuado que esté dispuesto a aceptar [12]; hacer evaluaciones si el servicio que da, supera sus expectativas de ganancia, aunando identificar la brecha a superar entre la evaluación de la calidad y los niveles de satisfacción del colaborador interno con respecto al servicio recibido [13]. En este sentido, la calidad del servicio al colaborador interno influye en su desempeño organizacional [14].

Por otra parte, los reportes sobre la percepción global del consumidor respecto a la calidad del servicio están positivamente asociados con la calidad del servicio del empleado, descrita en diferentes factores o dimensiones que determinan la calidad percibida [15]. Describen así, que el consumidor no sólo está interesado en lo que recibe como resultado del proceso de producción, sino en el proceso en sí mismo, aquilatando la responsabilidad social que despliega la empresa a través de los colaboradores de la organización.

Así, los sistemas de calidad de la gestión en toda 
institución, deben lograr una mejora de la percepción a la calidad del servicio tanto del usuario externo como del usuario interno y desarrollar estrategias para canalizar el conflicto organizacional como responsabilidad del ejecutivo, a la par de la preocupación de conocer el desempeño $\mathrm{u}$ otros procesos de la gestión del talento del recurso humano.

La percepción a la calidad de servicio de todo cliente con el objetivo del desarrollo sostenible mediante actividades de responsabilidad social es fuente de las percepciones en especificaciones de calidad de servicio [16], para el grupo de interés primario o nuclear de la institución [trabajador y su familia], el que está vinculado al estado de supervisor, ya que el mismo afectó directamente a dos de las variables mediadoras: el apoyo del supervisor y el conocimiento de los empleados sobre políticas amigables para la familia [17] en la conducta de todo usuario interno.

La percepción del servicio del colaborador interno es la significación valorativa mediante categorías, cuya connotación se debe a la cultura organizacional, que va adquiriéndose durante la socialización entre usuarios internos con respeto al sistema organizacional. La percepción de la calidad de servicio del colaborador interno es medida en 4 factores: la cooperación entre usuarios internos, liderazgo de gestión, valoración de su labor y la responsabilidad social institucional [4].

\section{B. Conflicto organizacional}

Es en este contexto socio laboral, escenario de interacción entre colaboradores y contexto psicosocial-ambiental de la institución, se plantea el constructo de Conflicto organizacional.

Planteando que el conflicto organizacional es una conducta, resultante de la interacción propia en todo contexto social que realizan los colaboradores, generada por la intencionalidad de obtener logros o satisfacer necesidades, ante la demanda por enfrentar situaciones o actividades por escasez de recursos o medios para su labor, diferencias o disyuntivas de enfoque o metodología para desarrollar actividades y funciones, también por la incongruencia entre los objetivos, valores, necesidades o expectativas personales con respecto a los del sistema organizacional [2] [7] y entre otros factores generadores o fuente del conflicto organizacional tales como, el que la gestión tiende soslayar, postergar o encubrir errores, anular o minimizar la participación en la toma de decisiones, así mismo por las desavenencias no concertadas entre actores en la gestión.

De ahí que, el conflicto es inherente a las organizaciones y al ser humano, haciéndose presente durante toda la vida, para el caso de los conflictos en el sistema social organizacional son direccionados a tener efectos positivos, constructivos generando la cohesión grupal por injerencia de la comunicación continua [18] lograda por el feedback organizacional o por lo contrario negativos, destructivos, conllevando frustración, pérdida de sinergia, originando barreras en la comunicación, afectando negativamente el performance de los usuarios internos de la organización.

En términos generales las interacciones socio laborales son fuente de la actitud que generan conflictos organizacionales, descritas en dos perspectivas: conflicto que lleva al cambio y a la mejora o conflicto que perturba y eleva los niveles de estrés laboral, comúnmente denominado conflicto funcional $\mathrm{y}$ conflicto disfuncional [19] [20]

Entre dichas fuentes del conflicto estarán las preferencias del CEO al jerarquizar actividades en base a motivaciones personales o de grupo, tal como, cuando da prioridad la distribución de los recursos o medios; como la formación de grupos que optan por el conflicto como una estrategia, a propósito de direccionar ganancias hacia la meta o lograr objetivos [21].

En esa perspectiva, también es fuente de la actitud, la intencionalidad de decidir a nivel individual o grupal el cambio de los términos del trato pertinente al objetivo institucional, restructuración o cambio organizacional que no es expresamente manifestada por la vía verbal o escrita, suscita el rumor, proclive a dejar al libre albedrio la formación de percepciones en base a categorías aprendidas que distorsionen el objetivo, dando paso evidente al conflicto tanto en usuarios internos como a la institución.

Las categorías con una dirección negativa hacia la fuente del conflicto organizacional son identificadas y aprendidas como objeto de actitud del usuario interno, de modo que, al superar el nivel de indiferencia o de tolerancia, pueda perturbar al colaborador durante el desarrollo de su actividad; generándose un entorno medioambiental laboral del que cabe señalar: "cuando el ambiente organizacional no tiende a satisfacer las necesidades del personal, puede generar conflictos, siendo el conflicto inevitable" Kelly, 1974, citado por [8] “(...) y un manejo evasivo de éstos afecta negativamente al desempeño organizacional de los colaboradores internos [22]; y puede también ser factible que se transforme en una fuente de efectividad organizacional" [8], ante la condición del cambio organizacional, el que, por implicar tensión, persistencia o frecuencia, elevará los niveles de estrés laboral e incidirá en el ambiente psicológico del grupo social, siendo potencial generador de cambio y estrategia en el proceso continuo del plan de mejora. Se hace más evidente en entornos cambiantes la presencia de conflictos organizacionales que puede retardar el progreso e incluso precipitar la desaparición de la organización [5], [23]

Por sus protagonistas, el conflicto organizacional se clasifica como un tipo de conflicto social, generado en un contexto social reglamentado, y por la cantidad de participantes, como un conflicto interpersonal o intergrupal. existiendo regulaciones específicas de los procesos y de los criterios de evaluación de los resultados en términos de competencias y particularidades propias para tener competitividad en el entorno complejo de instituciones del sector público. ([24], [19])

El modelo hipotetizado presenta una construcción en dos áreas: 1) Conflictos Individuales y 2) Conflicto del sistema organizacional [7], señalado también como niveles: individual y organizacional [18], a la que se suma la propuesta de tres dimensiones del conflicto organizacional observadas en las categorías: conflicto organizacional de escasez, conflicto 
organizacional interiorizado y conflicto organizacional emergente (Fig. 1).

La medición o manejo empírico de esta variable se basó en determinar el conflicto actitudinal, el conflicto organizacional registrando la dirección de la disposición, positiva o negativa, y su intensidad según la escala de respuesta ante estímulos propios del contexto socio-laboral, correspondientes a la primera etapa del conflicto social o de relación conflictiva subyacente en la organización [25] y la toma de posición sobre el desacuerdo existente, propio de la segunda etapa de cualquier conflicto social [25] a partir de la teoría del escalamiento y el desescalamiento del conflicto sobre el conflicto social.

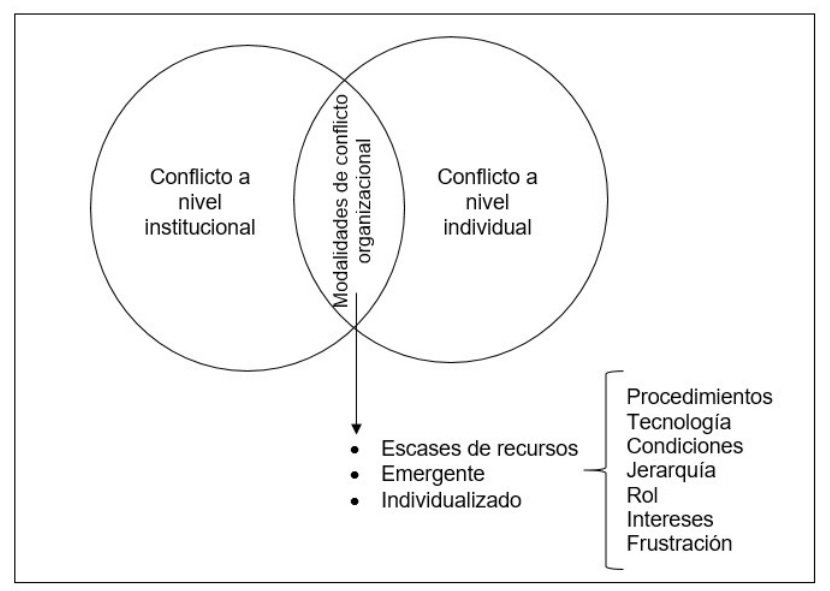

Fig. 1. Modelo de Conflicto organizacional: Tres modalidades específicas del conflicto organizacional de los dos niveles.

\section{Los conflictos individuales y conflictos del sistema organizacional}

El conflicto organizacional estudiado en dos áreas o niveles, que surge en diversos grados y modalidades a partir de interacción generada en los procesos de la actividad laboral, fuente real para posibilitar colisión de intereses entre ellos a procedimientos, recursos, tecnología, jerarquía, como sentimiento de frustración, conflicto de intereses, conflicto de rol, de manera personal o grupal [26] de la relación laboral entre los colaboradores, afectando el grado valoración y dirección de la percepción de los climas laborales y conductas en la organización institucional [27], como suelen originarse también por rasgos o características de personalidad según demandas o circunstancias personales en el contexto laboral [28].

Las demandas superiores a la capacidad individual producen sentimientos de frustración, los que actúan como fuentes generadoras de conflictos individuales, de una magnitud que rebasa el control emocional, debido entre otros a los siguientes factores, a) escasas habilidades sociales, b) conocimientos o competencias insuficientes y c) discusiones en cuanto al respeto a la responsabilidad del puesto de trabajo. De los tres factores mencionados, el vector principal en la socialización laboral, lo constituyen las habilidades sociales, entre ellas las habilidades de negociación [26], siendo de igual importancia el factor emocional, observado en el correspondiente control [29] en su rol de la evitación de incurrir en interpretaciones antagónicas o de tipo catastrófico, ante inconvenientes que en algunos casos sólo son aparentes, y fruto de la tensión ocasionada por una meta de alta tolerancia a la frustración, y en otras ocasiones, por la carga laboral o sobre control emocional o autocontrol [29] ante el servicio a usuarios externos, tales como la autoexigencia ante pacientes o estudiantes en condición continua, ha de conducir al estrés laboral al nivel de burnout [30].

La percepción está sujeta a la postura individual en la institución, y en independencia de la posición o rol que desempeñe aun cuando le confiere responsabilidades al usuario interno que de manera natural le provoca tensión, la que puede tener diversas respuestas saludables o no. Que puede sufrir distorsiones y expresarse en autosuficiencia o incapacidad, sea por soberbia o envidia, esta última, especialmente, cuando la posición de ejecutivo o directivo no legitima aportes o soslaya consulta, construyendo un contexto socio-laboral laxo, de confrontación entre sus integrantes, fuente de climas laborales descritos como contaminados [31].

El conflicto del rol formal en la organización puede formar parte de la fuente de conflicto, en casos en que la responsabilidad del ejecutivo se otorga sin autoridad vinculada a la competencia de la posición, o en la promoción a posiciones más elevadas, cuando se da un tipo de vinculación con compañeros $u$ amigos, quienes por dicha relación no lo aceptan en su jerarquía, de tal forma que, logra disociar su toma de decisiones [32].

En una institución en la que se dan estos comportamientos, ocurre que, por el contrario, el colaborador interno se sabe capaz o apto, pero se da la propensión a sentirse timado o engañado, al empleársele como "trampolín", y por ende, ante la exigencia del cumplimiento de las normas, se da paso a una fuente de conflictos personales en el sistema. Identificándose luego como conflictos organizacionales, activados ante deficiencias o intereses personales no valorado dentro de las unidades de la estructura institucional y la gestión, agravándose por la disposición a lograr su cometido generando conflictos personales y pretendiendo agudizarlo como alternativa o estrategia por propósitos de poder.

Así indica resultados que, un clima de apoyo moderaría de forma significativa y relevante la relación e influencia de la comunicación de funciones directivas y el conflicto de rol [33] otros muestran una relación positiva entre el conflicto de roles y el desempeño en el trabajo [34] donde la percepción de respaldo de la organización al desempeño de los supervisores, los hace sentirse empoderados por las relaciones con los subordinados [35] señalando que la ambigüedad del rol es un buen predictor [36] de los conflictos organizacionales emergentes.

Una estructura institucional débil o deficiente puede incluso pretender que, algunos conflictos se extiendan como una forma de aumentar la rivalidad, arguyendo la búsqueda de competitividad entre algunos trabajadores [37] $\mathrm{Y}$ aun cuando no existiera esta disposición en la gestión, o tuviera calidad en la gestión institucional, siempre existirán fuentes de conflictos 
que provengan de la estructura, y no de los individuos.

El conflicto del sistema organizacional atribuible a la estructura, se vincula con los elementos de la cultura organizacional elaborados deficientemente, o insuficientemente orientados, o diseñados en relación a las metas de la organización, pero, igualmente es la informalidad por la improcedencia o falta de aplicación de los principios de la administración [38] la fuente imparcial de conflictos y efectos negativos a la salud.

\section{Conflicto organizacional por escasez}

La limitación de recursos es un problema constante de las organizaciones públicas y privadas. Ante su solución elegida se complica por factores psicológico, entre ellos la motivación o por criterios de estrategia, lo que da lugar al tipo de conflicto organizacional por escasez, manifiesto en el mal empleo del recurso por parte de quien lo ha recibido y la consiguiente generación de percepción de inequidad. Dichos conflictos también tienen como componente afectivo producto de las experiencias previas de sus protagonistas, quienes han sufrido carencias o limitaciones para funcionar o realizar su actividad laboral, agudizado por la falta de prevención de un flujo continuo de aprovisionamiento. [39].

Sobre la institución recae la competencia de gestionar y proveer los recursos, previniendo la carencia o el desabastecimiento. Por su naturaleza, este tipo de conflicto remite principalmente a los conflictos en el sistema de la organización, atribuidos a la gestión.

\section{E. Conflicto organizacional interiorizado}

El conflicto interiorizado se genera a partir del propio usuario interno, debido a que las personas no reaccionan directamente al medio, sino que realizan previamente construcciones de significados personales [40], tal como lo dice la teoría adleriana, por su énfasis en la relevancia de los significados, propósitos, creencias y metas personales para la formación de actitudes, las que, en el caso de estar plenas de interés social propician estados de bienestar y de bajo estrés [41], una condición necesaria para el desarrollo de resiliencia y tolerancia ante la frustración [42]. Pero un déficit en la construcción del interés social conlleva poca preparación para cooperar en forma plena y la disposición a resaltar los defectos o errores de otras personas por una falsa percepción de lo que debe ser la superioridad.

En este caso se está haciendo referencia a que en el colaborador interno existen conflictos latentes durante su actividad en el sistema social organizacional. Las situaciones que se perciben como relación conflictiva subyacente son comportamientos de otros colaboradores, caracterizados por no ser acciones o productos de la gestión, como ocurre con la escasez de recursos o por ser emergentes ante divergencias en el sistema organizacional [43]. Las manifestaciones de este tipo de conflicto son demandas agresivas o pasivo agresivas hacia los diferentes niveles de posición de la estructura organizacional, sostenidas por su autopercepción de valía para desafiar incluso al sistema organizacional, promoviendo estrategias de apoyo a sus objetivos personales.

\section{F. Conflicto organizacional emergente}

Hace referencia al conflicto que surge ante el requerimiento de algún desafío, cambio, innovación o de tecnología en la organización, sea en algún sector específico o más bien general no compatible con principios o normas que, eventualmente se produzca por ausencia de instrucción o comunicación asertiva. Su surgimiento es el reflejo del tipo de afronte de la organización frente a las exigencias que debe encarar. Es tan propio del conflicto individual como del conflicto en el sistema de la organización.

Involucrarse en este tipo de conflicto por parte del usuario interno indica su preocupación por los aspectos de la gestión, en cualquier nivel de la estructura organizacional o etapa de los procesos de gestión de calidad. $\mathrm{O}$ a la deficiente aplicación de los principios de la administración, que al causar desorden despierta sentimientos de desasosiego, comportamientos individualistas. Con cualquiera de estas fuentes el clima laboral se convierte en desfavorable llevando a quejas, boicots, revanchas, denuncias pasibles de llevar al escalamiento del conflicto.

\section{G. El cliente o colaborador interno}

El cliente o colaborador interno es aquella persona que se desempeña dentro de la organización en diversa posición de la estructura, realizando actividades ya sean operativas, administrativas o ejecutivas [26]. Quien es proveedor o receptor de insumos o productos tangibles o intangibles, requeridos o necesarios para alguna de las etapas o fases del producto que se le demanda. Su importancia exige que, los sistemas de calidad de la gestión se traduzcan en procesos de mejora en pro de una cultura de calidad del servicio, avalada a través de una percepción favorable, no sólo por parte del usuario externo y particularmente del colaborador o usuario interno, donde el ejecutivo tiene como responsabilidad también de preocuparse de investigar además de su desempeño, el de indagar y conocer la percepción del servicio ofertado de su subordinado a sus pares o el inmediato superior en la estructura organizacional; identificando como categorizan y que atribuyen a la colaboración de sus compañeros, para cumplir con su labor. Y aunque, de hecho, la calidad total se apoya en la percepción del cliente externo [16] hoy por hoy, el objetivo del milenio está centrado en la responsabilidad social, cuyo grupo de interés primario o nuclear, lo constituyen el trabajador y su familia.

Ante este último aspecto, efectuar el análisis de los riesgos psicosociales, desde la investigación en psicología del trabajo y de las organizaciones, cumple un rol fundamental para la calidad de vida y humanización del trabajo como objetivo para la sociedad y empresas del siglo XXI, como parte de la responsabilidad social [44].

Lo señalado se confirma a través de reportes de estudios que consignan que la percepción global del consumidor sobre la calidad del servicio está directamente asociada con la calidad del servicio del empleado, descrita por diferentes factores o dimensiones que determinan la calidad percibida [45], [15]. Por ejemplo, el consumidor no sólo está interesado 
en lo que recibe como resultado del proceso de producción, sino en el proceso en sí mismo, valorando la responsabilidad social que despliega la empresa con sus colaboradores. De igual manera, se observa correlaciones significativas entre las dimensiones de la percepción del servicio del usuario interno con valores que van de $r>0,56$ al 0,64 , significativas al 0,001 en usuarios internos de PYMES [4].

\section{MetodologíA}

\section{A. Participantes}

Se contó con la colaboración de 378 participantes, que respondieron voluntariamente al cuestionario, de los cuales finalmente formaron parte del estudio sólo 303, por haber respondido a la totalidad las escalas y no tener distorsiones en las respuestas por aquiescencia; una muestra compuesta por trabajadores de instituciones públicas del ejecutivo, legislativo, judicial e instituciones de organismos descentralizados quienes dieron su consentimiento informado.

\section{B. Instrumentos}

La percepción de la calidad del usuario interno fue medida usando una escala compuesta por 18 ítems. Dicha escala mide la percepción del usuario interno a través de cuatro dimensiones: Cooperación entre usuarios internos, Liderazgo de la gestión, Valoración de su labor y Responsabilidad institucional [43]. Para las respuestas se usa una escala de cuatro puntos o grados, que van de total desacuerdo al total acuerdo. Los puntajes altos indican el mayor grado de valoración del aporte del usuario interno hacia los demás usuarios. El análisis psicométrico se efectuó con la estimación de la confiabilidad por consistencia interna, la evidencia de validez por la capacidad discriminante y el uso del análisis factorial confirmatorio para la estructura original de cuatro factores correlacionados indica un ajuste apropiado, 2 (146) = 260,61; CFI $=0,94 ;$ RMSEA $=0,037$ confirmación de la estructura factorial [43].

Por otra parte, la actitud hacia el conflicto organizacional fue medida con una escala compuesta por 12 ítems, correspondientes a tres factores o dimensiones: Conflicto por escasez, emergente e interiorizado, a nivel institucional e individual. Las respuestas son toman con una escala de cuatro puntos o grados, que van de total desacuerdo al total acuerdo. Los puntajes altos indican la disposición a iniciar un conflicto en la organización, a partir de doce afirmaciones que presentan la reacción ante situaciones que suelen generar discrepancias, mostrando que ha hecho ya el análisis del aspecto informativo y ha generado el objetivo de actuar para resolver el conflicto, haciendo uso de la cognición para determinar una relación conflictiva y la emoción, para a través de la emoción y generar sentimientos de indignación con deseos de reclamar, si se diera en la realidad. Puntúa a través de una escala de 4 grados, del total acuerdo al total desacuerdo.

Sus resultados psicométricos consisten en un adecuado ajuste a la estructura de tres dimensiones correlacionadas $\square 2$ $(51)=69,1 ; \mathrm{p}<0,047 ; \mathrm{CFI}=0,979 ; \mathrm{RMSEA}=0,033$.

\section{Procedimiento}

1. La recolección de datos se efectuó con estricta aplicación del consentimiento informado, de acuerdo con las pautas éticas de la investigación científica.

2. Los instrumentos fueron aplicados tanto en forma individual como colectiva.

\section{Resultados}

El procedimiento básico consistió en la evaluación del ajuste de los datos al modelo teórico propuesto se realiza mediante un análisis de ruta usándose el estimador de máxima verosimilitud. La evaluación del ajuste del modelo se realizó con el índice de ajuste comparativo (CFI) y el error cuadrático medio de aproximación (RMSEA). Se interpretan los valores $\geq 90$ en CFI y TLI como evidencia favorable de ajuste al modelo [46], así como de $\leq 0,08$ en RMSEA [47]. Para el análisis de confiabilidad se usó el análisis de consistencia el estadístico denominado Omega [48]. Este coeficiente es una alternativa ante las limitaciones ampliamente discutidas del coeficiente alfa [49] [50]. El software usado fue el IBM AMOS 24.

Como parte inicial del análisis se calcularon las correlaciones entre las dimensiones de las variables de estudio tal como se muestra en la Tabla 1. También se calcularon los coeficientes de consistencia interna omega, los que se encuentran en la diagonal de la tabla en mención, estos valores se encuentran entre 0,68 y 0,81 .

Junto a estos resultados se encontró que el coeficiente de correlación con la fórmula de Pearson es igual a $-0,206^{* *}$, significativo al 0,01 en una prueba de dos colas para la relación entre los puntajes totales de las escalas de Percepción de la calidad del servicio del usuario interno y actitud hacia el conflicto organizacional.

Finalmente, fue evaluado el modelo hipotetizado, encontrándose un adecuado ajuste a los datos, teniéndose este alrededor del criterio, $\square^{2}(8)=24,50 ; \mathrm{p}=0,002 ; \mathrm{CFI}=0,99$; TLI $=0,97$; RMSEA $=0,081$. El modelo explica el $49 \%$ de variabilidad en conflicto de escasez y $63 \%$ en conflicto emergente. En la Fig.2 se visualiza los coeficientes estructurales del modelo hipotetizado. 
TABLA I

CORRELACIONES, COEFICIENTE OMEGA Y DESCRIPTIVOS DE LAS DIMENSIONES DE PERCEPCIÓN DEL SERVICIO DEL USUARIO INTERNO Y CONFLICTO ORGANIZACIONAL

\begin{tabular}{|l|c|c|c|c|c|c|c|}
\hline Medición & $\mathbf{1}$ & $\mathbf{2}$ & $\mathbf{3}$ & $\mathbf{4}$ & $\mathbf{5}$ & $\mathbf{6}$ & $\mathbf{7}$ \\
\hline $\begin{array}{l}\text { 1. Cooperación entre usuarios } \\
\text { internos }\end{array}$ & {$[0,79]$} & & & & & & \\
\hline 2. Liderazgo de la gestión & 0,61 & {$[0,81]$} & & & & & \\
\hline 3. Valoración de su labor & 0,84 & 0,68 & {$[0,78]$} & & & & \\
\hline 4. Responsabilidad institucional & 0,78 & 0,63 & 0,70 & {$[0,68]$} & & & \\
\hline 5. Conflicto por escasez & $-0,10$ & $-0,24$ & $-0,06$ & $-0,01$ & {$[0,67]$} & & \\
\hline 6. Conflicto interiorizado & $-0,05$ & 0,01 & 0,11 & $-0,06$ & 0,62 & {$[0,73]$} & \\
\hline 7. Conflicto emergente & $-0,17$ & $-0,19$ & $-0,06$ & $-0,16$ & 0,63 & 0,77 & {$[0,71]$} \\
\hline$M$ & 13,2 & 12,2 & 13,4 & 11,0 & 9,4 & 12,2 & 10,3 \\
\hline$D E$ & 3,7 & 4,9 & 3,6 & 3,8 & 4,5 & 4,5 & 4,4 \\
\hline
\end{tabular}

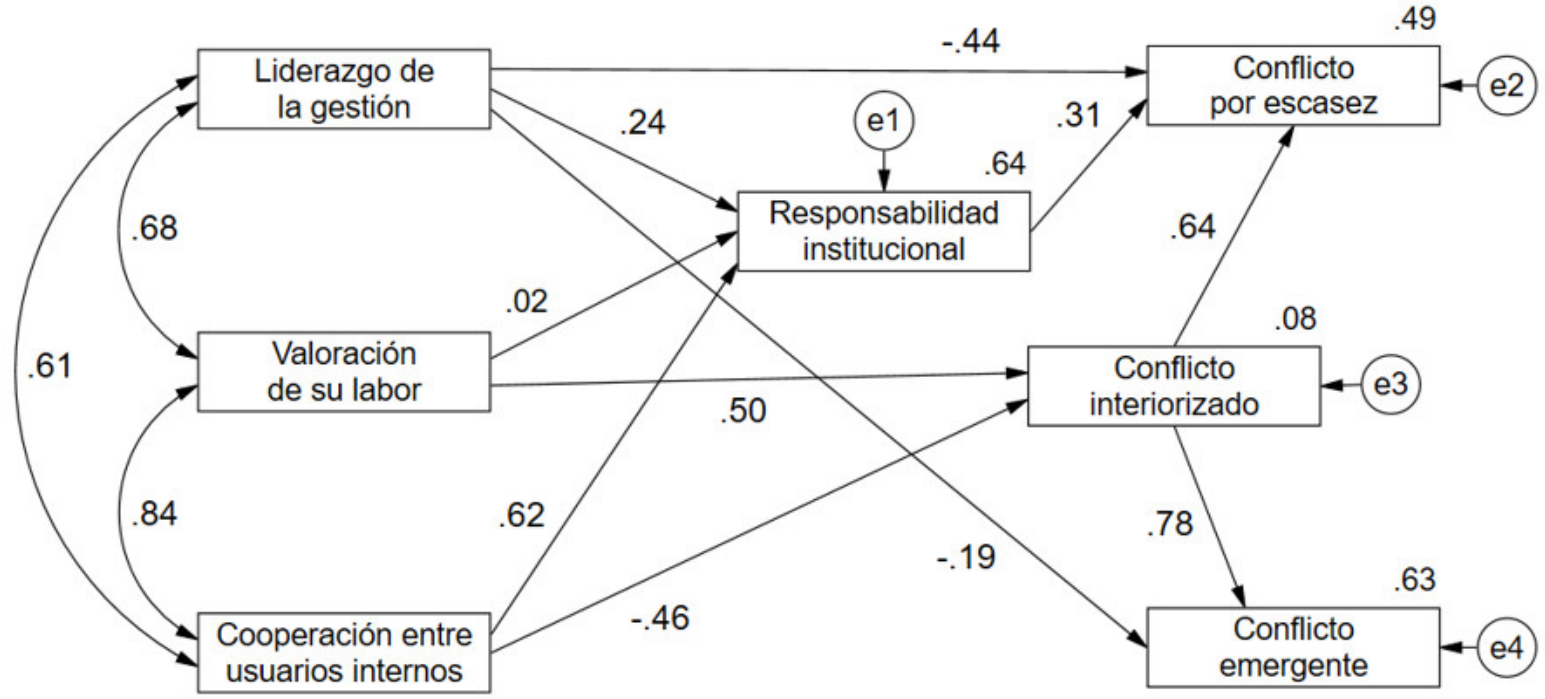

Fig. 2. Coeficientes estandarizados del modelo de conflicto organizacional y percepción del servicio del usuario interno 


\section{V.DISCUSIÓN Y CONCLUSIONES}

En la naturaleza de las organizaciones subyacen los conflictos que pueden presentarse tanto en su estructura como en sus procesos. Las actitudes con las cuales los afronten los miembros de la organización pueden volverse funcionales o disfuncionales, es decir, convertirse en retos para actualizar los potenciales de los trabajadores o colaboradores internos o tornarse en obstáculos que conlleven a la paralización, estancamiento e incluso liquidación de la organización.

Partiendo de esta premisa, se propuso un modelo teórico según el cual la funcionalidad en la gestión en las organizaciones consiste en la oposición entre la percepción favorable del servicio del usuario interno y la actitud desfavorable hacia el conflicto en la organización. La percepción que se construya en torno a la calidad del servicio brindado entre colaboradores está acompañada de la disposición a la confianza y a la colaboración entre compañeros, las cuales servirán para centrarse en el problema antes que en las personas y su actitud hacia el conflicto se caracteriza por ser proactiva y resolutiva.

Un hallazgo importante con respecto a que factores son el pivote de estas correlaciones inversas, es el liderazgo de la gestión, dado que, al considerarlo óptimo según la percepción del colaborador interno, va a conllevar la generación de un clima laboral saludable, mediante la cooperación entre usuarios internos, y evite el deterioro en las relaciones interpersonales.

Otro hallazgo relevante es la elevada correlación existente entre la valoración de su labor con la cooperación entre usuarios internos, nos indica la significación que tiene el proporcionar o atender con calidad a un requerimiento, dado que incide en la percepción de la valoración de su labor e implica estima personal; así mismo, la incidencia de la relación entre la gestión del liderazgo con la valoración de la labor, responsabilidad institucional y la cooperación entre usuarios internos, confirma la incidencia que tiene el ejercicio del liderazgo en la gestión para la formación de percepciones de las relaciones entre colaboradores internos en dirección positiva.

Los resultados son lo suficientemente consistentes para generar estrategias de intervención aun en el caso del conflicto interiorizado o de tipo individual, este último alejado de la intervención directa del líder o de la gerencia. Es el caso particular de la única excepción a las correlaciones de dirección inversa, como es la relación entre la valoración de la labor con el conflicto interiorizado, se observa que este último es inaccesible a la acción directa del liderazgo de la gestión, no obstante, se encuentran que la cooperación entre colaboradores internos le permite actuar de manera indirecta.

Se observa que, las relaciones entre los factores investigados describen la naturaleza de las instituciones públicas debido al carácter burocrático de estas, al ser un espacio en el que las interacciones son fuentes de conflictos organizacionales. Dichos conflictos son gestionables para obtener niveles óptimos de funcionalidad, si es que se tiene en cuenta que, un resultado funcional o disfuncional se dará a partir de la percepción de la calidad del servicio que exista entre los colaboradores internos, aun cuando por tratarse de la gestión burocrática se esperan resultados predecibles por el tipo de dirección, que se caracteriza por ser de jerarquía vertical [51] por las normas, entre ellas, las que regulan los conflictos sociales en la organización [25]; y así también por las relaciones sociales, las normas de gestión, los procesos productivos, todos ellos delimitados por parámetros sociales construidos por la organización, que dan vinculación simbólica y emocional con la organización [52], [53]

Es importante señalar que las investigaciones sobre la calidad del servicio que se le brinda al colaborador interno son escasas, aun cuando se enfatiza en la relevancia del capital social organizacional para que las organizaciones sean sostenibles [54]. En circunstancias en que prima la inestabilidad y la incertidumbre en las organizaciones, cuando la principal fortaleza que tienen es el colaborador interno [55].

\section{AGRADECIMIENTOS}

Por el financiamiento a la UNMSM (Vicerrectorado de Investigación y Posgrado), IPPSI de la Facultad de Psicología, la participación voluntaria de los colaboradores de las diversas instituciones públicas. Así mismo al Ps Ronald Castillo Blando por el procesamiento y análisis de datos de la investigación.

\section{REFERENCIAS}

[1]CEPAL. "Agenda 2030 y los Objetivos de Desarrollo Sostenible Una oportunidad para América Latina y el Caribe". Naciones Unidas. Chile. 2015

[2]J. Hernández y A. Mosquera. "La gerencia de los conflictos en las organizaciones modernas: estrategias de acción". Centro de Investigación de Ciencias Administrativas y Gerenciales, Vol. $8 \mathrm{~N}^{\circ} 2$, pp. 77-87. 2011. ISSN: 1856-6189

[3]O. Regalado, R. Allpacca y L. Baca. Endomárketing: estrategias de relación. Lima, Universidad ESAN. 2011

[4]M. Paredes, H. Hernández y A. Chung. "Relaciones de confianza y percepción al servicio del colaborador interno para la responsabilidad social en pequeñas empresas de parques industriales de Lima Metropolitana". Industrial Data, Vol. $19 \mathrm{~N}^{\circ}$ 2, pp. 21-29. 2016. ISSN: 1810-9993. doi: http://dx.doi.org/10.15381/idata.v19i2.12812

[5]S. Sarmiento y M. Paredes. "Percepción de la calidad de servicio del usuario interno en una institución pública". Industrial Data, Vol. $22 \mathrm{~N}^{\circ}$ 1, pp. 95-112. 2019. ISSN: 1810-9993. doi:, https://doi.org/10.15381/

[6]E. Mikkelsen y S. Clegg. "Conceptions of Conflict in Organizational Conflict Research: Toward Critical Reflexivity". Journal of $\begin{array}{lllll}\text { Management. } & \text { Vol. } & 28 & \mathrm{~N}^{\circ} & 6 .\end{array}$ https://doi.org/10.1177\%2F1056492617716774

[7]R. Hodgetts y S. Altman. Comportamiento en las organizaciones. México, McGraw Hill. 1994

[8]M. Rahim [2002]. "Toward a theory of managing organizational conflict". The International Journal of Conflict Management. Vol. $13 \mathrm{~N}^{\circ} 3$, pp. 206-235. 2002. ISSN: 1044-4068

[9]L. Bohórquez. "Diseño de un modelo de auto-organización para la solución de las crisis en las organizaciones empresariales". Entre Ciencia E Ingeniería. Vol. $11 \mathrm{~N}^{\circ} 21$, pp. 14-21. 2019. ISSN: 2539-4169 https://doi.org/10.31908/19098367.3269

[10] G. Balmori y J. Flores. "MECSI: Modelo para evaluar la calidad del servicio interno". Innovaciones de Negocios. Vol. $11 \mathrm{~N}^{\circ} 2$, p. 191. 2014. ISSN: 2007-1191.

[11] J. Carlson. El momento de la verdad. España, Ediciones Díaz, Santos S.A. 1991.

[12] V. Zeithaml, L. Berry and A. Parasuraman [1993]. "The Nature and Determinants of Customer Expectations of Service". Journal of the Academy of Marketing Science. Vol. $21 \mathrm{~N}^{\circ}$ 1, p. 11. 1993. ISSN: 15527824. 
[13] T. Vu Van [2015]. "Determinants of customer expectations of service: implications for fostering customer satisfaction". 2014 Proceedings of ISER-Science Plus International Conference. Diciembre 2014.

[14] Sh. Srivastava1 y G. Prakash. "Internal Service Quality: Insights from Healthcare Sector". Journal of Health Management. Vol. $21 \mathrm{~N}^{\circ} 2$, pp. 294-312. 2019. http://dx.doi.org/10.1177/0972063419835127.

[15] K. Govender. "Managing service quality by managing the service encounter". Cape Town. University of Cape Town. Tesis doctoral en Filosofia. 1998. https://open.uct.ac.za/handle/11427/9574.

[16] B. Font. "El marketing de la calidad en el sector turístico". Esic Market, pp. 59-93. 2000. ISSN: 0212-1867.

[17] K. Ramadoss y M. Lape. "Supervisor Status, Health and Organizational Commitment: Mediating Role of Support and Family-friendly Policies among Information Technology Professionals in India". Journal of Business and Management Sciences. Vol. $2 \mathrm{~N}^{\circ}$ 1, pp. 1-9. 2014. ISSN: 2333-4533.

[18] M. Valbuena, R. Morillo, M. Montiel y J. Hernández. "Gerencia estratégica y los conflictos organizacionales". Multiciencias, Vol. 12, pp. 270-276. 2012. ISSN: 1317-2255.

[19] S. Robbins y T. Judge. Comportamiento organizacional. México, Pearson. 2013.

[20] K. Boulding. "Las tres caras del poder”. Barcelona, Paidós. 1993.

[21] R. Barrera. Negociación y Transformación de Conflictos: Reto entre Escasez y Bienestar. Editorial Serviprensa. Guatemala. 2004.

[22] MG. Muthoni y S. Muathe. "Organizational Conflict Management: The All-Important Public Universities Performance Strategy Under Neglect in Kenya". European Scientific Journal. Vol. $17 \mathrm{~N}^{\circ}$ 12, p. 181. 2021. ISSN: 1857-7431. https://doi.org/10.19044/esj.2021.v17n12p181.

[23] A. Klitan, A. Mclay y T. Pilli. "Managing conflict in organisational change". International Journal of Agile Systems and Management. Vol.9 No2. 2016.2 ISSN: https://dx.doi.org/10.1504/IJASM.2016.078575

[24] C. Fernández y E. Polanco. "Manejo de conflicto en instituciones públicas de educación superior del estado Zulia". Revista de Artes y Humanidades UNICA. Vol.10 N³. 2009. ISSN: 1317-102X.

[25] L. Kriesberg [1975]. Sociología de los conflictos sociales. México, Ed. Trillas. 1975

[26] M. Paredes. "Las Habilidades Interpersonales para la Negociación según el Estilo de vida y el Nivel ocupacional en Empresas Productoras del Perú". UNMSM. Lima. Tesis de Doctorado en Psicología. 2010.

[27] A. Garcés. [2004]. Medición del Clima Organizacional. Madrid, Aguilar. 2004.

[28] K. Davis y J. Newstrom. El Comportamiento Humano En El Trabajo. Comportamiento organizacional. México, McGraw-Hill. 1991.

[29] R. Bar-On. "Emotional and social intelligence. Insights from the emotional quotient inventory". R. Bar-On, \& D. Parker, en The handbook of emotional intelligence: Theory, development assessment and application at home, school and in workplace, pp 363-388. San Francisco, JosseyBass. CA. 2000.

[30] V. Olivares. "Laudatio: Dra. Christina Maslach, Comprendiendo el Burnout". Ciencia \& trabajo. Vol. $19 \mathrm{~N}^{\circ}$ 58, pp 59-63. 2017. ISSN: 0718-2449. doi: https://dx.doi.org/10.4067/S0718-24492017000100059.

[31] C. Pulido. "Construcción, validación y aplicación de una prueba que mide el Clima Organizacional de organizaciones empresariales privadas y su aplicación para la psicología de la Salud". UNMSM. Lima. Tesis de Maestria. 2002.

[32] A. Lucas, P. García y S. Llano. Sociología de las organizaciones: Influencia de las tecnologías de la información y la comunicación. Madrid, Fragua. 2013.

[33] P. Diaz-Fúñez, V. Mañas-Rodríguez y V. Pecino-Medina. "El efecto modulador del clima organizacional en la influencia de la comunicación de funciones directivas sobre el conflicto de rol". 2016.

[34] B. Babin and J. Boles. "The effects of perceived co-worker involvement and supervisor support on service provider role stress, performance and job satisfaction". Journal of Retailing. Vol. $72 \mathrm{~N}^{\circ}$ 1, pp 57-75. 1996. ISSN: 0022-4359.

[35] L. Shanock and R. Eisenberger. "When supervisors feel supported: Relationships with subordinates' perceived supervisor support, perceived organizational support, and performance". Journal of Applied Psychology. Vol. [91], pp 689-695. 2006. ISSN: 0021-9010.

[36] R. House and J. Rizzo [1972]. "Role conflict and ambiguity as critical variables in a model of organizational behavior". Organizational Behavior and Human Performance. Vol. $7 \mathrm{~N}^{\circ}$ 3, pp 467-505. 1972. ISSN: 0749-5978. doi: https://doi.org/10.1016/0030-5073-90030-X.
[37] Harvard Business School Publishing Corp. "Los pros y contras de la competencia entre empleados". Diario El Financiero Costa Rica. 21 de octubre de 2017

[38] G. Requejo [2017]. "Relación entre la percepción de la informalidad interna con el estrés laboral en trabajadores de Lima Metropolitana res de organizaciones de Lima". UNMSM. Lima. Tesis de Maestría. 2017.

[39] Y. González. "El conflicto organizacional: una solución constructiva". Revista Pensando Psicología. Vol. $6 \mathrm{~N}^{\circ}$ 4, pp.11-27. 2010. ISSN: 2382-3984.

[40] C. Méndez. Clima organizacional en Colombia. El IMCOC: Un método de análisis para su intervención. Bogotá, Universidad del Rosario. 2006.

[41] R. Miller and D. Dillman. [2016]. "Does Adlerian Theory Stand the Test of Time: Examining Individual Psychology from a Neuroscience Perspective". The Journal of Humanistic Counseling, Vol. $55 \mathrm{~N}^{\circ} 2$, pp 111-128. 2016. ISSN: 2159-0311. doi:10.1002/johc.12028.

[42] H. Wagnild and H. Young. "Development and psychometric evaluation of the Resilience Scale". Journal of Nursing Measurement. Vol. $7 \mathrm{~N}^{\circ} 2$, pp. 165-178. 1993. ISSN: 10613749.

[43] M. Paredes y H. Hernández. "Estructura factorial de la escala de percepción de la calidad del servicio del usuario interno para instituciones públicas". Universidad Nacional Mayor de San Marcos. Lima. 2018. En proceso de arbitraje.

[44] J. Peiró, J. [2009]. "Psicología de las organizaciones: Investigación, Formación y Práctica Profesional". en Serie Profesores Honorarios ed. Centro de Producción. Lima, Editorial e Imprenta de la UNMSM.

[45] M. Guzmán. "Dimensión Interna De La Responsabilidad Social Empresarial Desde La Óptica De La Gestión De Recursos Humanos". Saber. Vol. $28 \mathrm{~N}^{\circ}$ 4. 2016. ISSN: 2343-6468.

[46] P. Bentler [1990]. "Comparative fit indixes in structural models". Psychological Bulletin. Vol. $107 \mathrm{~N}^{\circ}$ 2, pp 238-246. 1990. ISSN: 00332909. doi:10.1037/0033-2909.107.2.2.238.

[47] R. MacCallum, M. Browne and H. Sugawara. "Power analysis and determination of sample size for covariance structure modeling". Psychological Methods, Vol. $1 \mathrm{~N}^{\circ}$ 2, pp 130-149. 1996. ISSN: 1082989X. doi:10.1037/1082-989X.1.1.130.

[48] R. McDonald [1999]. Test theory: A unified treatment. Mahwah, N.J, Erlbaum Associates. 1999.

[49] E. Cho. "Making reliability reliable: A systematic approach to reliability coefficients". Organizational Research Methods, Vol. 19 N $^{\circ} 4$, pp 1-32. 2016. ISSN: 1094-4281. doi:10.1177/1094428116656239.

[50] K. Sijtsma. "On the use, the misuse, and the very limited usefulness of Cronbach's alpha". Psychometrika, Vol. $74 \mathrm{~N}^{\circ}$ 1, pp. 107-102. 2009. ISSN: 0033-3123 doi:10.1007/s11336-008-9101-0.

[51] C. Flink. "Multidimensional conflicto and organizacional performance". American Review of Public Administration. Vol. $45 \mathrm{~N}^{\circ} 2$, pp.182-200. 2015. https://doi.org/10.1177\%2F0275074013490825.

[52] J. De Miguel. La organización como construcción social Representación organizacional. Madrid, Universidad Autónoma de Madrid. 1999.

[53] M. Paredes, J. De Miguel, M. Carbajal y M. Laguna. "Construcción social de la comunidad para el desarrollo del callejón de Huaylas, Caraz". Revista de Investigación en Psicología. Vol. $7 \mathrm{~N}^{\circ} 1$. pp. 81102. 2004. ISSN: 1609-7475. https://doi.org/10.15381/rinvp.v7i1.5140

[54] J. Vargas. "Capital social organizacional, base de la sustentabilidad organizacional para el desarrollo ambiental y económico: el caso de las micro y pequeñas empresas en la explotación del tule typha spp de la Laguna de Zapotlán”. Revista CIFE: Lecturas De Economía Social. $\begin{array}{llllll}\text { Vol. } 12 & \mathrm{~N}^{\circ} & 16 . & 2010 . & \text { ISSN: } & 2248-4914\end{array}$ https://doi.org/10.15332/s2248-4914.2010.0016.07

[55] E. García. "Capital social, conocimiento y efectividad organizacional". Enl@ce Revista Venezolana de Información, Tecnología y Conocimiento. Vol. $11 N^{\circ}$ 3. 2014. Pp. 33-48. ISSN: 1690-7515. 


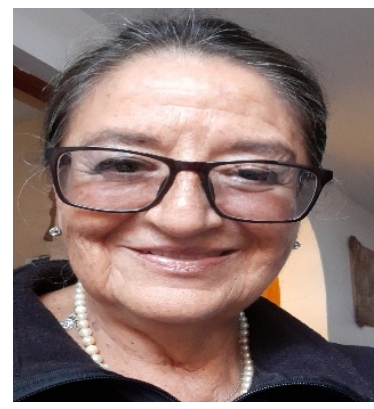

Mildred Paredes Tarazona. Estudió en la Universidad Nacional Mayor de San Marcos, Lima-Perú; Psicóloga, especializada en Psicología Organizacional y Doctora en Psicología. Magíster en Administración con mención en Comercio InternacionalC; Becada por el Gobierno de Israel al curso "Desarrollo Comunitario y Organización de Cooperativa Rural" Tel Avid- Israel (1985); Profesora en el Doctorado del Programa de Intercampus, MadridEspaña (1999), Profesora Tutora del Programa "San Marquinos para el Perú" en la Universidad Harvard (junio 2017). Docente Principal e investigadora, docencia desarrollada desde 1990 a la fecha. Publicaciones de investigaciones por más de 25 años en el ámbito de la psicología organizacional, estudios de psicología social- económica y modelos aplicativos para comprender el comportamiento y gestión de la organización, directora de la Escuela de Psicología Organizacional y de la Gestión Humana (febrero 2019- actualidad). Directora del Centro de Responsabilidad social de la Facultad de Psicología (octubre 2013- abril 2017). Miembro Docente Principal - Facultad de Psicología de la Asamblea Universitaria de la UNMSM. (2016-2020). Presidenta de la Comisión Permanente de Fiscalización de la UNMSM. (2016-2017). Asesora de la Dirección General de Posgrado, VRIP, UNMSM (noviembre 2016- abril 2018), ORCID: https://orcid.org/0000-0002-96320927

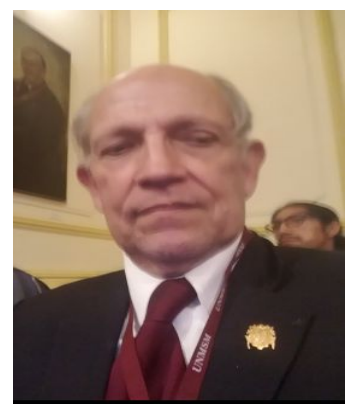

Héctor Manuel Hernández Valz. Nació en Tarapoto, San Martín, Perú, en 1995 obtuvo el título profesional de Psicólogo en la UNMSM, posteriormente, en 2019 el grado de Magister en Psicología con mención en Psicología educativa por la UNMSM. Ejerce profesionalmente como docente asociado en pregrado en la UNMSM. También tiene publicación de diversos artículos científicos, publicados en diferentes revistas de investigación. El Mg. Hernández figura en la lista de investigadores de Concytec y es miembro del grupo de investigación Vínculos Interdependientes y Contextos Medio Ambientales Saludables en el Trabajo (VINCTRAB). También es miembro de la orden del Colegio de Psicólogos del Perú. ORCID: https://orcid.org/0000-0002-3122-7633

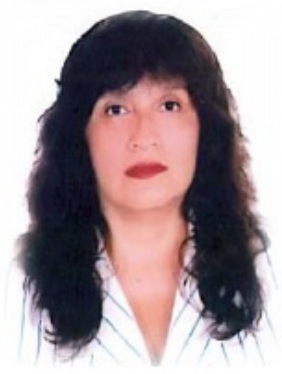

Carmela Malaver Soto. Nació en Lima, Perú, en 2006 obtuvo el título profesional de Psicóloga por la Universidad Nacional Mayor de San Marcos. En el 2017 concluye sus estudios de Maestría en Psicología con mención en Psicología Organizacional en la Universidad Nacional Mayor de San Marcos. Ex becaria para la Diplomatura "Desarrollo Integral y Prevención de la Violencia Juvenil" por la Organización Panamericana de Salud y del Programa de Formación Profesional de Países Iberoamericanos por la Universidad de Salamanca. Docente auxiliar de la UNMSM, post Jefa de la Oficina de Calidad Académica y Acreditación de la Facultad de Psicología de la UNMSM. Miembro titular del Instituto de Investigaciones de Psicología y de los Grupos de Investigación Vínculos Interdependientes y Contextos Medio Ambientales Saludables en el Trabajo y Psicología, Sociedad, Violencia y Bienestar. Las temáticas de interés a investigar están relacionadas a liderazgo y responsabilidad social, empresas saludables, bienestar y violencia en las organizaciones. ORCID: https://orcid.org/0000-0002-0464-8918

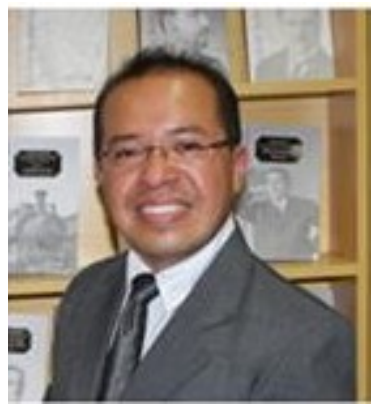

Alfonso Ramón Chung Pinzás. Nació en Lima, Perú, en 1999 obtuvo el título profesional de Ingeniero Industrial por la Universidad Ricardo Palma, posteriormente en el 2005 el grado de Magíster en Ingeniería Industrial con mención en Producción Industrial por la Universidad Nacional Mayor de San Marcos (UNMSM) y el 2012 el grado de Doctor en Ingeniería Industrial por la UNMSM. Ejerce profesionalmente como docente principal de pre y posgrado en la UNMSM, además de ser director de la Escuela Profesional de Ingeniería Textil y Confecciones de la Facultad de Ingeniería Industrial de dicha universidad, así como editor de la revista científica Industrial Data. También es autor de tres libros, así como de diversos artículos publicados en revistas indexadas. Los intereses de investigación del autor están relacionados al clima laboral, prospectiva, modelamiento y docencia universitaria. El doctor Chung es investigador RENACYT, miembro de los grupos de investigación de Gestión del Conocimiento y Vínculos Interdependientes y Contextos Medio Ambientales Saludables en el Trabajo, también es miembro de la orden del Colegio de Ingenieros del Perú. ORCID: https://orcid.org/0000-0002-8073-2396. 